\title{
PROOF-OF-CONCEPT INVESTIGATION OF ACTIVE VELCRO FOR SMART ATTACHMENT MECHANISMS
}

\author{
Joseph W. Clement* and Diann E. Brei ${ }^{\dagger}$ \\ Department of Mechanical Engineering \\ The University of Michigan \\ Ann Arbor, MI 48109-2125
}

\begin{abstract}
A Smart Attachment Mechanism (SAM) possesses the ability to attach two surfaces and effect relative motion between the surfaces upon command. This paper introduces a new SAM technology called Active Velcro which exhibits these capabilities. The conceptual design and its operation are explained along with a brief discussion of several key design issues that govern its architecture. Proof-of-concept prototypes were fabricated at two scales: one at a large-scale $(26 \times 22.5 \times 6 \mathrm{~cm})$ using off-the-shelf components, and a second at a reduced-scale $(0.55 \times 0.63 \times 0.21 \mathrm{~cm})$ utilizing stereolithography. In bench-top tests, the prototypes demonstrated planar translation and rotation capabilities while retaining the part being manipulated thereby proving the Active Velcro concept.
\end{abstract}

\section{Introduction}

An active surface is comprised of several actuators arranged in a dense array. Such a surface has many advantages over single point actuators. By utilizing the combined effort of the actuators, much like the manner in which the cilia of the respiratory tract translate material or the legs of the centipede generate locomotion, the active surface can generate motions over greater distance with greater force than achievable with a single point actuator while maintaining a high resolution. Complex motions incorporating both translation and rotation also become possible, opening the door for many applications. Several active surfaces have been developed to provide active position and orientation control on both micro- and macro-scales.

On the micro-scale, MEMs batch processes allow for the fabrication of highly dense actuator arrays. For

\footnotetext{
*Graduate Student, Department of Mechanical

Engineering and Applied Mechanics

${ }^{\dagger}$ Assistant Professor, Department of Mechanical

Engineering and Applied Mechanics

Copyright (C) 2001 The American Institute of

Aeronautics and Astronautics Inc. All rights reserved.
}

example, Suh et al. ${ }^{1}$, Ebefors et al. ${ }^{2}$ and Ataka et al. ${ }^{3}$ used thermally activated polyimide benders to provide accuracy in the $5-10 \mu \mathrm{m}$ range in translation, with Suh's system demonstrating rotation capabilities as well. Applications requiring higher precision and a smaller stroke were considered by Langlet et al. ${ }^{4}$ Their Scratch Drive electrostatic actuators provided translation control over a $50 \mu \mathrm{m} \times 40 \mu \mathrm{m}$ range with 25 $\mathrm{nm}$ resolution to meet the needs of applications such as micro fiber optic coupling. Iizuka and Fujita ${ }^{5}$ developed a micro-conveyance system capable of teleoperation in clean rooms for the assembly of MEMS scale devices. Their device consisted of a small aluminum plate incorporating four permanent magnets that was levitated above a superconductor. Motion was generated through magnetic propulsive forces created by the interaction of the permanent magnets located on the plate with a grid of active energized electrodes. Nakazawa et al. ${ }^{6}$ devised a similar system utilizing small in-plane electromagnetic coils to generate the necessary levitation as well as propulsion forces to create a system capable of planar translation at speeds up to $30 \mathrm{~mm} / \mathrm{s}$. Konishi and Fujita ${ }^{7}$ developed an active surface utilizing electrostatically valved micro air jets to both levitate and propel a mover at translation speeds greater than $5 \mathrm{~mm} / \mathrm{s}$ over a distance of $1 \mathrm{~mm}$. Early work by Bohringer et al. ${ }^{8,9}$ utilized torsional microresonators to develop a system capable of planar translation and rotation with enough force to manipulate silicon wafers.

For the manipulation of larger items, several macroscale active surfaces have been developed. Tadokoro et al. ${ }^{10}$ designed an active surface utilizing $2 \mathrm{~mm}$ long Ionic Conducting Polymer gel Film actuators (ICPF) to transport soft objects such as biologic organs without damage. Their surface operated in a liquid bath demonstrating translation speeds up to $0.62 \mathrm{~mm} / \mathrm{s}$. Yim et al. ${ }^{11}$ developed a modular self-reconfigurable robot comprised of 1-DOF degree of freedom $5 \mathrm{~cm}^{3}$ cubic modules capable of manipulating objects as well as locomotion with multiple distinct gaits to handle varying terrain and obstacles. Luntz et al. ${ }^{12,13,14}$ 
developed an array of 2 inch diameter roller wheels to autonomously position and orientate parcels using both open-loop and closed-loop control.

While many of the current active surfaces have successfully demonstrated precise position and orientation control of objects, none of them provide any retention force between the two surfaces. All of the devices rely on gravity to prevent the surfaces of the devices from separating. This limits usage to stable nonmoving environments experiencing gravitational forces. The active positioning of objects onboard moving vehicles such as ships, aircraft, or ground vehicles becomes difficult. Operation in the reduced gravity of space becomes an impossibility.

It is desirable to have a smart attachment mechanism (SAM) that can provide the translation and rotation capabilities of the previously mentioned active surfaces, while retaining the part being manipulated. Potential applications for SAMs exist in both military and industrial fields. On the micro-scale, SAMs could be could be used for micro fabrication, part sorting, or precision optics. Scaled up versions of a SAM could be used to transport supplies and compactly store them on ships, provide connection and fine adjustment of very large deployable structures for space optics, or be used on the exterior of inflatable bodies so that radar or optical systems could be actively positioned and reconfigured for adjustable aperture size and different cluster formations. Another example is satellite docking where the smart attachment mechanism could capture and position an incoming satellite, which would repair damage, replenish expendables, or upgrade components.
This paper presents a fundamental SAM concept nicknamed "Active Velcro" (because of its similar passive engagement method compared with traditional Velcro $^{\mathrm{TM}}$ ). The overall system architecture and its operation are described along with a discussion of several key design issues. Proof-of-concept prototypes were fabricated at two scales to verify the concept's ability to generate translation and rotation while retaining the part being manipulated.

\section{Active Velcro Design}

Active Velcro is a two surface mechanism designed to offer full planar translation and rotation capabilities while providing a positive retention force between the surfaces (Figure1). One surface serves as the host, comprised of a highly redundant hexagonal array of active prongs, as well as the actuation, control, and power systems. The second surface serves as a guest, comprised solely of a sparsely patterned array of inactive posts. The posts and prongs have a unique topology such that the surfaces passively connect to each other when they come into contact. Once in contact, the host surface is activated to position and orientate the guest surface to the desired location.

\section{System Operation}

Engagement of the two surfaces occurs through a snapfit connection, which entraps each inactive post of the guest surface within a prong grouping on the host surface. This prong grouping consists of three prongs arranged in a triangular formation (Figure 2a). By straining Shape Memory Alloy (SMA) wires located at the center of alternating prong groupings, the prongs can be actuated to position and orient the guest surface. The SMA wires are attached to the prongs through a unique flexure mechanism (Figure 2b), which transmits

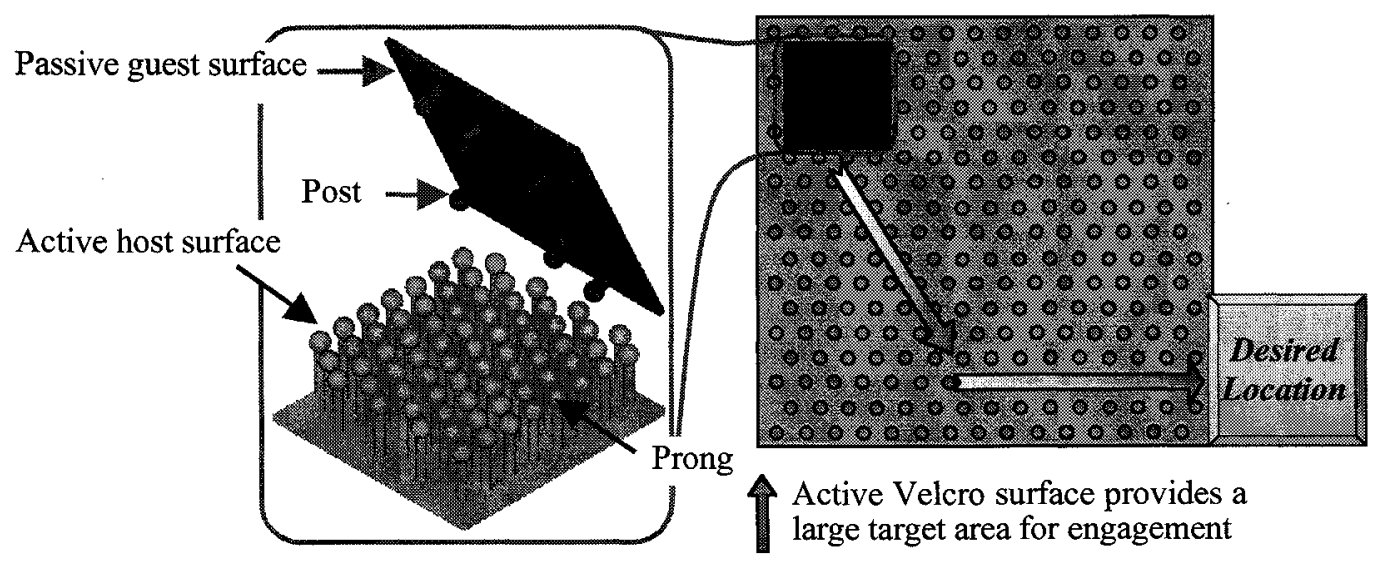

Figure 1: Active Velcro Concept Overview. Active Velcro consists of two surfaces: one inactive guest surface with a sparse arrangement of posts and an active host surface with a densely distributed array of actuated prongs. The host surface actively inches the guest surface to the desired position and orientation. 
the work of the individual SMA wire to the three adjacent prongs.

Activating a single SMA wire causes its three adjacent prongs to deflect inward towards the center of the prong grouping in a grasping action. This motion alone is incapable of providing relative motion of the two surfaces. However, relative motion can be generated by activating multiple adjacent SMA wires simultaneously (Figure 4). When two adjacent SMA wires are activated, both individual prong groupings will attempt to deflect inward with the same grasping action, but the shared prong will move according to the resultant force vector provided by the combined work of the two adjacent energized SMA. This resultant vector is oriented perpendicular to a line connecting the two energized SMA wires. Two basic gaits are used to generate motion in the guest surface depending on the location of the post.

The first gait, depicted in Figure 3a, occurs when a post is located inside a prong grouping containing an SMA wire. If this SMA wire and two of its adjacent neighbors are activated simultaneously, there will be two shared prongs. These two prongs, which had originally entrapped the post, will move perpendicular to the row of activated SMA wires and out of the way of the advancing post. The post is advanced by the unshared prong which still deflects to the center of its prong grouping; thereby, pushing the post through the two shared prongs to the next prong grouping. This completes one translational step.

After this translation, the post is located in a prong grouping without an SMA wire and the second gait is

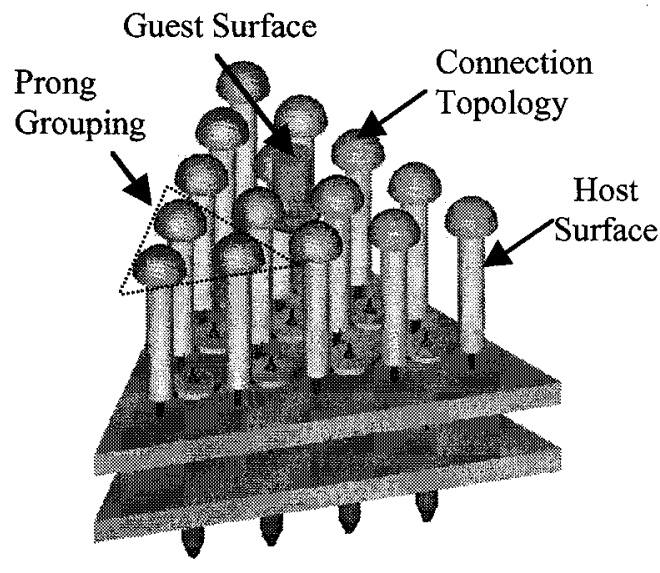

a) Host Surface Detail employed (Figure 3b). For this gait, only two SMA wires are energized which creates one shared prong. This shared prong pushes the post through the two unshared prongs to the next prong grouping. This new grouping has a SMA wire and requires the first gait; thus, the cycle repeats. This process can be repeated multiple times to inch the guest surface to the desired location in a saw-toothed motion and can be applied to one or many posts on the guest surface. By altering the timing and directions of translation applied to multiple posts of the guest surface, rotation can be achieved.

With only a single post being translated, the speed of the translation is primarily limited by the time that must be allotted between steps to allow the SMA wires to cool before the next step can begin. If a larger redundant array of posts is used, increased translation speed may be possible since sequential translations can be achieved without requiring any SMA wire to cool between steps. This is accomplished by energizing only enough SMA wires to directly push a subset of the total engaged posts forward. Because all posts are rigidly connected together, the remaining posts are pushed (or pulled) along into the next grouping even though their respective prong groupings were not energized. Translation can continue immediately by heating a different subset of cooled SMA wires; thereby, eliminating the slow cooling response of the SMA wires from the translation sequence. Operation in this phased activation manner allows an SMA wire to cool for several steps while other SMA wires are performing the translation. Therefore, translation speed is now only a function of the heating response time of the SMA wires, which increases the potential speeds by approximately an order of magnitude (depending on the

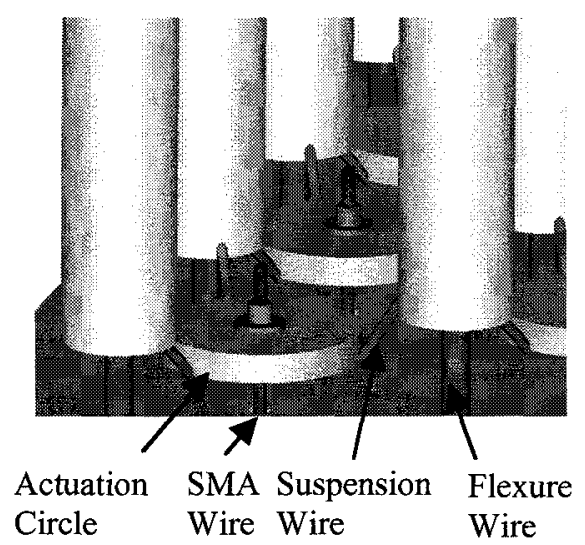

b) Flexure Mechanism

Figure 2: Host Surface Detail. The host surface consists of a densely patterned array of actuated prongs. The prongs are activated by SMA wires located at the center of alternating prong groupings. When a given SMA is energized, the deflection of the SMA wire acts on the center of the actuation circle, which in turn distributes the work to the three attached prongs. This causes the three prongs to flex inward creating a grasping action. Complex prong motions can be generated by simultaneously activating multiple SMA wires. 


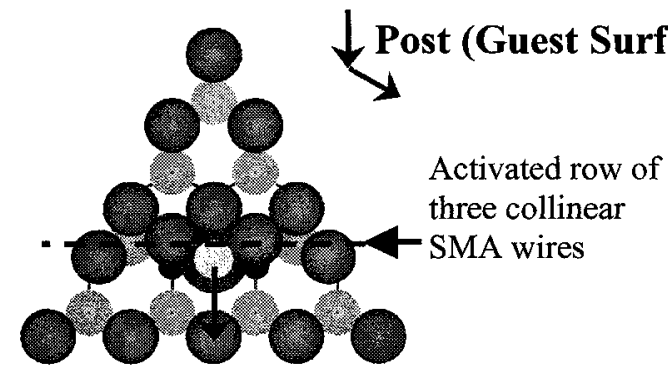

a) Unshared prong pushes post

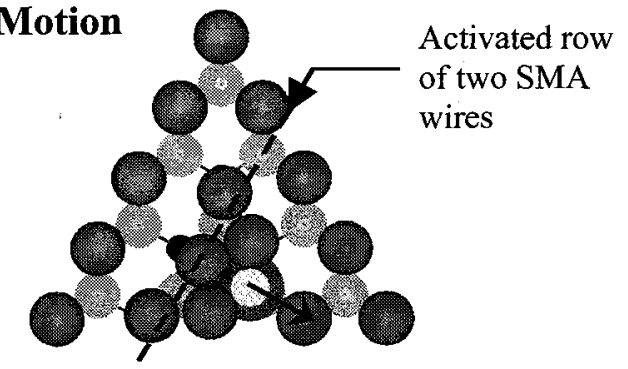

b) Shared prong pushes post

Figure 3: Global System Operation. Translation proceeds in a saw-tooth manner. The guest surface moves orthogonal to the energized row of SMA wires.

SMA characteristics) when compared to situations requiring complete SMA cooling between steps.

\section{System Design Issues}

Developing an active surface with full planar motion capabilities while providing positive retention between the surfaces raises two significant design issues: the host surface pattern and the design of the post/prong connection topologies.

\section{Surface Pattern}

The development of a pattern of host surface prongs well suited to both translation and rotation is a critical aspect of the Active Velcro design. The current host surface consists of triangular prong groupings, which at first does not seem well suited to translation due to the necessity of a saw-toothed translation motion as opposed to purely linear motion that would be offered by a four-pronged system. While the rectangular pattern of prongs that arises from using a four-pronged system has the advantage of direct linear motion, it reduces system resolution and increases actuator demands. For given post and prong diameters, the center-to-center distance between prongs is $29 \%$ smaller for the three-pronged system than it is for the four-pronged system (Figure 5a). Therefore, a 29\% improvement in the system resolution (defined as the smallest translation displacement / step) can be achieved with the three-pronged system without decreasing the size of the individual components of the device. Additionally, 29\% less actuator deflection is required to achieve each translation increment reducing the overall size and power requirements of the surface.
- Enetgizing a single SMA wire causes the three adjacent prongs to bend inward toward the center of the grouping.

- When adjacent SMA wires are energized, the resultant prong motion ( creates a path for the advancing post.
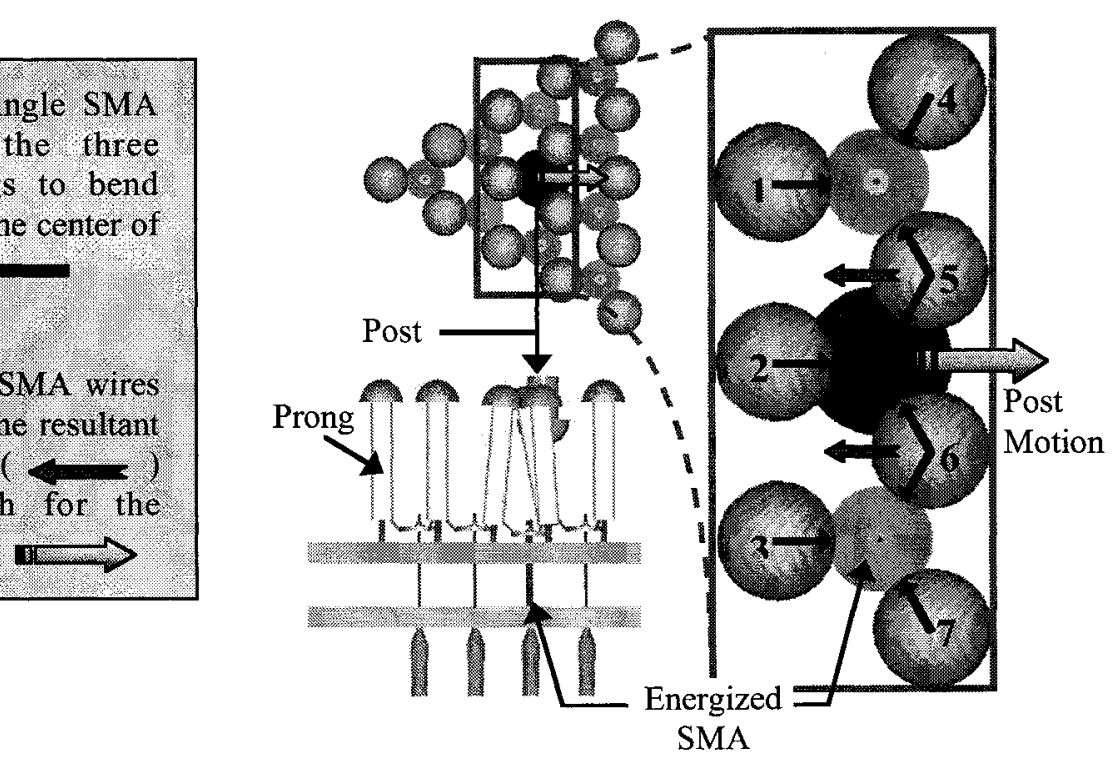

Figure 4: Local Prong Operation. If a post is located directly above a SMA wire, it can be translated to the right of the figure by energizing a vertical row of three adjacent SMA wires. In this case, the three prongs on the left side of the figure (prongs $1,2,3$ ) would all deflect towards the right with prong 2 actually pushing directly on the post. Prongs 4 and 7 would both deflect towards their respective attached and energized SMA wires. Prongs 5 and 6 are each acted upon by multiple SMA wires and therefore their motion is given by the resultant force vector created by energized wires (towards the left side of the figure). As a result of the individual prong motions, prong 2 pushes the post between prongs 5 and 6 to complete the translation of one step.

3

American Institute of Aeronautics and Astronautics 


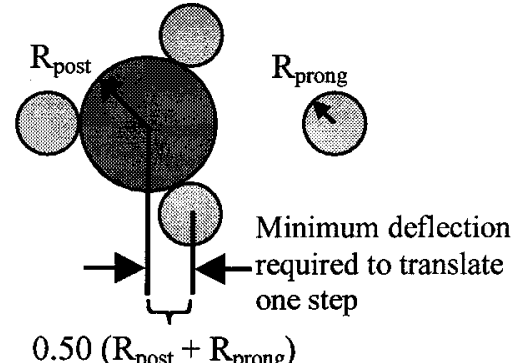

Three-Pronged System

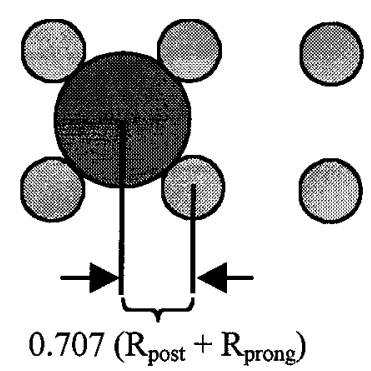

Four-Pronged System

a) Three-Pronged vs. Four-Pronged System

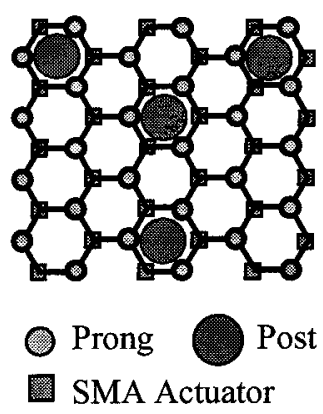

b) Hexagonal Repeat Unit (Three-Pronged System)

Figure 5 Three-Pronged System Properties: a) For given post and prong diameters, the three-pronged system results in a $29 \%$ improvement in system resolution with a corresponding $29 \%$ decrease in required actuator deflection when compared to a four-pronged system. b) The repeat unit of this array is a hexagonal cell in which three corners of the hexagon are occupied by prongs and the remaining three corners are occupied by SMA wires. The hexagon is the largest order regular polygon that can be arrayed without leaving irregular gaps in the pattern.

The three-pronged system also leads to an overall surface pattern better suited to rotation than the fourpronged system. For rotation, the system of prongs would ideally be patterned in such a way, that concentric circular pathways exist about any single point, allowing the guest surface to smoothly rotate about any given point on the host surface. With a fourpronged system, there exists a pattern of concentric squares about any given point in the array.

In contrast, the three-pronged system provides concentric rings of hexagonal pathways about a given location in the array. The hexagonal of prongs offered by the three-pronged system is visible in Figure $5 \mathrm{~b}$ where the repeat unit is a hexagonal cell in which three corners of the hexagon are occupied by prongs and the remaining three corners are occupied by SMA wires. The hexagon is the largest order regular polygon that can be arrayed without leaving irregular gaps in the pattern. For example, if regular octagons are arrayed, there will exist square shaped spaces at alternating sides of each octagon (a common household tiling pattern). These spaces would result in areas in which engagement would be impossible between the guest and host surfaces. Thus, for an arrayed system of regular polygons, a concentric ring of hexagonal pathways provides the best approximation to the ideal circular pathways. As such, the three-pronged system provides an increased potential for successful rotation than the four-pronged system or any other arrayed regular polygonal system.

\section{Connection Topology}

The second important design issue for Active Velcro is the topology selection for the engaging post and prongs, since this establishes the defining retention property of the Active Velcro concept. For a successful connection, there are a number of important design issues that must be addressed:

- When the guest surface post approaches the host prong system, the post should be guided into the center of a prong grouping instead of bouncing off the surface.

- Post engagement should require a minimum of force and occur passively so that the system doesn't always have to be powered.

- The force required to separate the surfaces should be large enough to prevent premature separation of the two surfaces.

- Any topology that is chosen must not impair relative motion of the two surfaces. Thus, in the concept presented in this paper, the topology must allow the post to slip between prongs during translation and rotation of the guest surface.

Fortunately, the connection forces (engagement, retention) and required forces for motion generation (translation, rotation) can be modified independently by altering key features of the connection topology. The surfaces most directly affecting connection and motion are depicted in Figure 6 . The lead surfaces of both the post and prongs determine the engagement force. For example, elongating the lead surface of the prongs from a spherical to a conical shape reduces the engagement force without affecting either the retention or required translation forces. The trailing surfaces of both the post and prong primarily determine the retention force. For example, increasing the slope of the prong's trailing surface reduces the retention force without affecting engagement or translation forces. The support columns and diameters of the connecting heads of both the post 


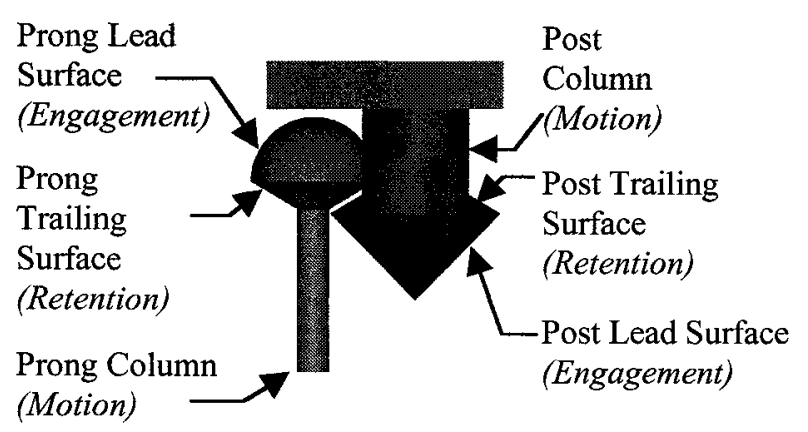

Figure 6: Sample Connection Topology. The surfaces most directly affecting engagement, retention, translation, and rotation can be independently tailored.

and prong primarily determine the required force for translation/rotation. For example, decreasing the diameter of the support column for the guest surface post with respect to a given prong head diameter decreases the required forces for translation because the post can now easily slip through the channels in the host surface array.

A variety of connection topology prototypes with lead and trailing surfaces ranging from spherical to conical to tetrahedral topologies were fabricated using the stereolithography process and tested on the basis of engagement and retention force. Engagement forces ranged from 2.9 to $22.6 \mathrm{~N}$ with retention forces ranging from 1.8 to $10.1 \mathrm{~N}$. While the study was simplistic and not all-inclusive, it verified that the engagement and retention forces could be tailored independently and helped to guide the selection of the post and prong connection topologies to the bulb/conical design depicted in Figure 6.

\section{Large-Scale Prototype Test Bed}

A large-scale prototype was originally designed and constructed to demonstrate the motion generation capabilities of the Active Velcro surface. The system was developed as a basic proof-of-concept and was not optimized for minimal power consumption, speed of motion, or maximum motion resolution. However, the prototype is highly adjustable and easily serviceable because it is composed of discrete parts. These features facilitated the large-scale prototype being an effective original test bed for examining translation and rotation techniques

\section{Large-Scale Prototype Fabrication}

The large-scale prototype was fabricated using off-theshelf components chosen based on the availability of materials and ease of construction. The host surface was constructed of a hexagonal array of 75 prongs mounted to a $13 \mathrm{~mm}$ thick polyethylene board (Figure 7). The prongs were spaced $26 \mathrm{~mm}$ apart, thus forming a hexagon with $130 \mathrm{~mm}$ side lengths.
The prongs were made from hollow $6.35 \mathrm{~mm}$ diameter PVC tubing that was cut to $50 \mathrm{~mm}$ in length. To form the connection topology, wooden caps of $13.3 \mathrm{~mm}$ diameter and $5.6 \mathrm{~mm}$ in height were attached to the top of each prong. To allow adjustment of the overall prong height and flexure mechanism amplification factor, steel music wire of $0.81 \mathrm{~mm}$ diameter was inserted into the bottom of each prong and affixed using set screws through the prongs. The music wire served as a flexure hinge when the system is fully assembled, allowing the prongs to pivot when actuated. By inserting the exposed end of the music wire into the polyethylene base, the prong was mounted.

To form the prong groupings, actuation circles were used to connect groups of three prongs. The actuation circles were fabricated from a $17.5 \mathrm{~mm}$ Delrin rod with a $3.5 \mathrm{~mm}$ center drilled hole. The rod was diced into 2.5 $\mathrm{mm}$ thick circles and three axi-symmetric grooves were machined into the bottom into which three steel wires were fitted. The free ends of the steel wires were attached to the bottom of three adjacent posts, suspending the actuation circle in the center of the prong grouping. This assembly serves to transfer to work of the SMA wires to the prongs.

A second $13 \mathrm{~mm}$ thick polyethylene board was used to serve as a lower anchoring point for the SMA wire. Holes of $5.5 \mathrm{~mm}$ diameter were drilled through both the top and bottom base to allow the SMA wire to pass through. The $75 \mathrm{~mm}$ on-center separation between the lower and upper base was necessary to provide an adequate SMA wire length to achieve translation.

A total of 61 SMA wires were strung from the actuation circles through the lower base. The SMA wire was held in place at the actuation circles using the leadholding mechanism from a $0.3 \mathrm{~mm}$ mechanical pencil and with a pin vise at the lower base. The $0.38 \mathrm{~mm}$ diameter Flexinol ${ }^{\mathrm{TM}}$ SMA wire was purchased from Dynalloy, which was rated for a maximum pulling force of 2000 grams, with a transition temperature of $90^{\circ} \mathrm{C}$. The active length of SMA wire for the large prototype was $82 \mathrm{~mm}$.

Two representative guest surfaces were constructed. The first consisted of a single Delrin post with a $24 \mathrm{~mm}$ diameter conical end topology mounted to a $142 \mathrm{~mm}$ square Plexiglas plate, thereby representing a simple guest surface. Similarly, a second guest surface with three identical posts was fabricated to more accurately represent an array of posts on the guest surface. 

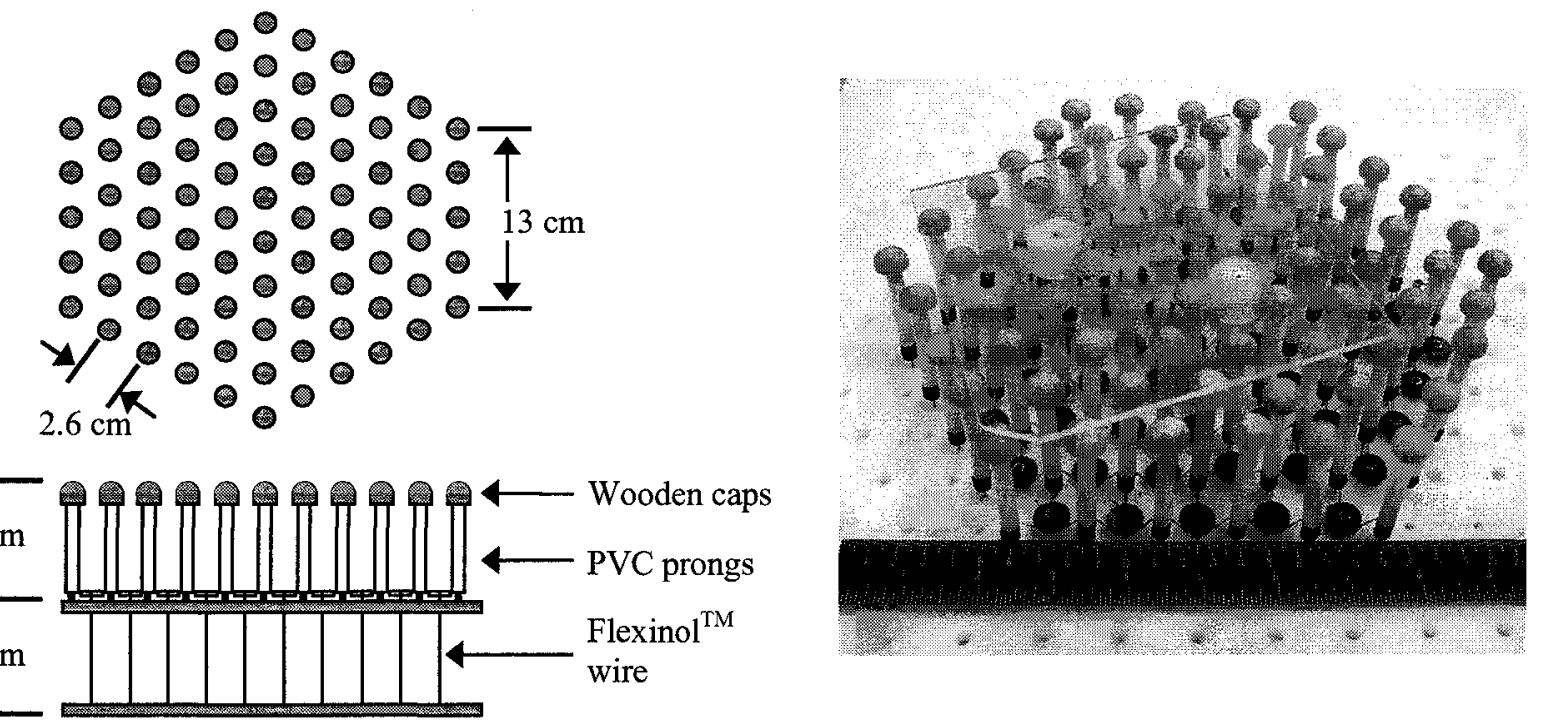

Figure 7: Large-Scale Prototype. A large-scale prototype was fabricated using off-the-shelf components to prove the motion generation capabilities of Active Velcro.

\section{Experimental Procedure and Results}

The SMA wires were energized by shorting the output of a Samlex PSA-310 power supply across the subset of SMA wires determined by the gait and desired guest surface motion. Resistive heating raised the temperature of the SMA wires above the transition temperature, thereby causing the SMA wires to contract.

Planar translations were successfully demonstrated using the large-scale prototype with both single-post and three-post guest surfaces (Table 1). The lengths of translations were limited only by the size of the host surface (single-post tests) and the number of SMA wires integrated into the host surface (three-post tests).

In addition, planar rotation has been demonstrated using a two-post guest surface. Rotation testing was performed in two manners. First, the guest surface was pivoted about one of the posts. For this fixed center-ofrotation testing, the prongs engaging the pivot post were not deflected while the prongs engaging the second post were deflected. It was not necessary to apply an external load to the pivot post to prevent it from moving during testing. This fixed center-ofrotation testing yielded a maximum repeatable rotation

Table 1: Performance Metrics for the Large-Scale Prototype

\begin{tabular}{|l|l|}
\hline Step Size & $17 \mathrm{~mm}$ \\
\hline Force (per post)* & $0.2 \mathrm{~N}$ \\
\hline Linear Speed & $3.7 \mathrm{~mm} / \mathrm{s}$ \\
\hline Input Power (per wire) & $4.9 \mathrm{~W}$ \\
\hline
\end{tabular}

* Estimated metric of approximately 40 degrees. Sixty degrees of rotation has been obtained, but was not repeatable due to excessive interference between the moving post and the host surface prong array above 40 degrees. This is a function of the surface pattern and the selected prototype dimensions. A refined surface design is currently being developed to achieve full rotation by investigating the impact of individual prong design and host surface pattern on the interference levels encountered during rotation. In the second test, the pivot point location was allowed to vary. During this variable center-of-rotation testing, the pivot point was varied from one post to the other as well as points in between. For rotation about points other than a post location, the prongs engaging both posts were deflected simultaneously. The variable center-of-rotation testing yielded a full 360 degrees of rotation.

For these tests, power consumption metrics were calculated based on the application of $2.75 \mathrm{Amps}$ to the wires. Under typical room temperature conditions, this results in heating/cooling times of approximately $0.7 / 9.0$ seconds. The voltage is based directly on the resistance of the wire multiplied by 2.75 Amps, yielding a required input power of $4.9 \mathrm{~W}$ per wire.

The step size of $17 \mathrm{~mm}$ was measured directly off the prototype. It is representative of the system resolution, indicating the minimum controllable translation increment. A large step size was used for the prototype to ease construction. Average linear translation speeds of approximately $3.7 \mathrm{~mm} / \mathrm{s}$ were obtained when translating a single post guest surface with an applied current of $2.75 \mathrm{~A}$ to the SMA wires. Speed measurements were determined by examining a digital 
video recording taken during experimentation. Because of the hexagonal prong array, a true linear translation is not possible. As a result, the quoted linear speed is calculated based on the linear distance between the starting and ending points of translation and does not include the extraneous distance traveled due to the sawtoothed motion.

To estimate the force transmitted to the guest surface, an approximation of the losses through the flexure mechanism was necessary. The flexure mechanism has been assumed only $50 \%$ efficient; therefore, only half of the usable work generated by the SMA is transferred to the guest surface. This is believed to be a conservative estimate. Using this flexure efficiency, coupled with the leverage ratio between the tip of the prong and the SMA wire, a force of $0.2 \mathrm{~N}$ (per post) is obtained. The total force applied to a guest surface is therefore the number of engaged posts times $0.2 \mathrm{~N}$.

\section{Discussion}

While the large-scale prototype is considerably larger than the final system should be for most applications, there are a number of important observations that can be made. The repeatable planar translation and rotation capabilities demonstrated the viability of the motion generation method on the large-scale and the promise of Active Velcro. The motion generation was repeatable using a hand built prototype with off-the-shelf components despite flaws in the construction, such as differences in the height and spacing of the prongs, asymmetries in the actuation circle and its three suspension lines, and variations in the overall stiffness of each prong. While the errors in construction have not been quantified, the repeatable translations and rotation obtained despite the errors reveal an insensitivity of the system design to flaws in construction.

Because the Active Velcro prototype is activated with macro-scale SMA wires, a significant power draw is incurred, with the requirement of $4.9 \mathrm{~W}$ (per wire). As the device is intended to be used in a redundant manner, many wires will be energized at a given time increasing the power demands. For example, if four guest surface posts were simultaneously pushed, twelve SMA wires would need to be simultaneously heated, requiring approximately $60 \mathrm{~W}$ of power. This is significantly more power than the smaller scale MEMS devices require, even in the case of thermally actuated active surfaces such as that of Bohringer et al. ${ }^{15}$, which can require in excess $4 \mathrm{~W}$ for the entire field of actuators. Yet, the overall displacement of the Active Velcro prototype is on the macro- not the micro-scale. In addition, it is important to note that power consumption will fall as the size of the SMA wire is decreased. Even with the relatively high power requirements, successful operation of the large-scale prototype has been achieved with a power source as small as eight AA batteries.

The translation speed of $3.7 \mathrm{~mm} / \mathrm{s}$ equates to approximately 0.3 translation steps per second, or 0.3 Hz. However, testing on the same 15 mil diameter SMA wires revealed a much lower $0.1 \mathrm{~Hz}$ bandwidth under similar room temperature conditions with no forced airflow ( 0.7 seconds heating, 9 seconds cooling). Therefore by integrating the SMA wires into the distributed Active Velcro surface, a three-fold increase in effective surface bandwidth was achieved. It is important to note that this performance increase is not due to the phased activation approach, although it has a similar effect. In this case, a given SMA wire may be energized on sequential steps but the wire need only partially cooled between these sequential steps. In contrast, the phased activation approach prevents any given wire from being energized on sequential steps thereby eliminating the need to cool a wire at all between steps which could increase translation speed to as high as $18 \mathrm{~mm} / \mathrm{s}$.

\section{Reduced-Scale Unified-Host Test Bed}

Lessons learned from experimentation with the largescale prototype test bed were utilized to design and fabricate reduced-scale prototypes with a single-piece upper host surface. The reduced-scale unified-host prototypes were designed with three primary objectives in mind:

- Ensure that the connection topology and motion can be combined together without impeding each other as the scale is decreased.

- Improve the construction of the prototype for a higher degree of precision and accuracy while simplifying assembly by reducing the part count.

- More accurately represent the final Active Velcro design by reducing the scale of the prototypes.

\section{Reduced-Scale Prototype Fabrication}

The reduced-scale prototypes were fabricated with a 3D-Systems SLA-250/40 stereolithography machine using Somos 8110 resin (Young's modulus $\sim 317 \mathrm{MPa}$, flexural modulus $\sim 310 \mathrm{MPa}$, elongation to failure $\sim 27 \%$ ). After the part was created, it was cleaned using a Branson 3200 Ultrasonic cleaner to remove any uncured resin. The layered building process inherent to stereolithography produces a very rough surface finish on the prototypes, which increases the engagement force and impedes motion due to excessive friction. To improve this finish, liquid resin was hand applied to the 

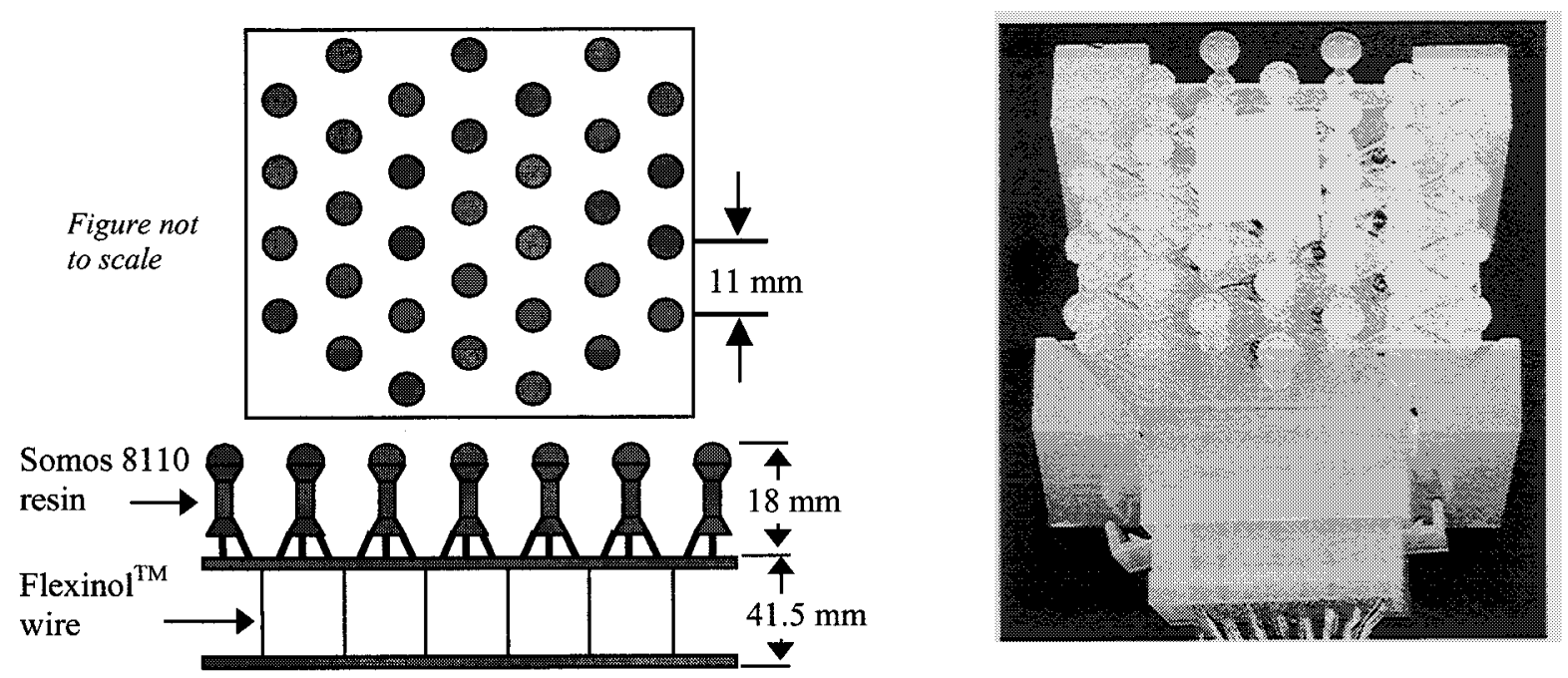

Figure 8: Reduced-Scale Prototype. The reduced-scale prototype was fabricated with stereolithography using Somos 8110 resin at approximately $1 / 3$ the scale of the large-scale prototype. The prototype has successfully demonstrated translation capabilities while retaining the manipulated surface.

connection elements of the cleaned prototype. The prototype was then cured for 15 minutes in a $3 \mathrm{D}$ Systems Post-Cure Apparatus (PCA). A second coat of liquid resin was applied to the connection elements and the prototype was cured for 60 additional minutes.

The reduced-scale prototype was built at approximately $1 / 3$ the scale of the large-scale prototype. The host surface was fabricated in two pieces: the upper surface containing the prongs, connection topology, flexure mechanism, and the backbone / the lower surface acting as an anchoring point for the SMA wires. A separate base was utilized for testing purposes only and could have easily been fabricated along with the upper base in a single piece. The separate lower base was attached to the upper host surface using adjustable spacers, which were used to rapidly alter the active length of the SMA wire. As with the large-scale prototype, the leadholding mechanism from a mechanical pencil was used to attach the SMA wire at the actuation triangle. The wires were secured beneath the base with $7.2 \mathrm{~mm}$ collars.

Figure 8 shows the resulting reduced-scale prototype. The host surface measured $55 \times 62.5 \mathrm{~mm}$ and contained 33 prongs spaced in an array similar to that of the largescale prototype. Each prong grouping consisted of three $18 \mathrm{~mm}$ tall prongs spaced $11 \mathrm{~mm}$ apart in an equilateral triangular pattern. The actuation triangle was suspended by three $1.5 \mathrm{~mm}$ diameter suspension lines. The lower surface was mounted $37.5 \mathrm{~mm}$ below the upper surface, yielding an active SMA wire length of $41.5 \mathrm{~mm}$. All testing was performed using a onepiece single-post guest surface. The post consisted of a $8.3 \mathrm{~mm}$ diameter, $6 \mathrm{~mm}$ tall support column with a
$9.5 \mathrm{~mm}$ diameter, $5 \mathrm{~mm}$ tall conical end topology represented in Figure 6.

\section{Experimental Procedure and Results}

By activating the reduced-scale prototype in an identical manner as large-scale prototype, repeatable translations of a single-post guest surface were achieved. Again, the number of sequential translation was limited only by the size of the prototype. A step size of $6.35 \mathrm{~mm}$ was utilized to demonstrate about a factor of three reduction in scale with respect to the large-scale prototype (Table 2). Translation speeds up to $2.8 \mathrm{~mm} / \mathrm{s}$ were achieved, approximately $75 \%$ of the large-scale prototype's speed, while providing approximately 2.67 times more translation resolution. Power consumption, when compared to the large-scale prototype, was reduced by $77 \%$ to $1.1 \mathrm{~W}$ per wire with the application of 0.9 A to each SMA wire.

\section{Discussion}

The reduced-scale prototype demonstrated the ability to fabricate the prongs, connection topologies, flexure mechanism, and backbone in a single piece while improving the accuracy and symmetry in the prototype. Because the stereolithography process is capable of rapidly fabricating complex three-dimensional structures, the construction of a single piece upper host

Table 2: Performance Metrics for the ReducedScale Prototype

\begin{tabular}{|l|l|}
\hline Step Size & $6.35 \mathrm{~mm}$ \\
\hline Force (per post)* & $0.1 \mathrm{~N}$ \\
\hline Linear Speed & $2.8 \mathrm{~mm} / \mathrm{s}$ \\
\hline Input Power (per wire) & $1.1 \mathrm{~W}$ \\
\hline
\end{tabular}

* Estimated metric

3

American Institute of Aeronautics and Astronautics 
surface was possible. This significantly reduced assembly time and insured that every prong was evenly spaced and aligned perfectly, something that was difficult to achieve with the hand fabricated large-scale prototype. With the reduced-scale prototypes, rotations were not possible due an insufficient number of prong groupings on the host surface. While it is possible to fabricate a reduced-scale prototype capable of rotation, the host surface array was kept small to reduce fabrication times and minimize distortion of the prototype during testing due to the transverse loadings generated by the SMA wires.

The translation speed of the reduced-scale prototype was approximately $75 \%$ of that of the large-scale prototype. This reduction stems from the smaller step size of the reduced-scale prototype, $6.35 \mathrm{~mm}$, compared to $17 \mathrm{~mm}$ for the large-scale prototype. Therefore, each cycle of the SMA wire produces only $6.35 \mathrm{~mm}$ of deflection, or approximately $37 \%$ of the deflection obtained per cycle with the large-scale prototype.

However, the smaller $200 \mu \mathrm{m}$ ( 8 mil) SMA wires can be cooled quicker due to their reduced thermal mass (1 second heating, 2.2 second cooling) thereby increasing an individual wire's bandwidth to approximately 0.3 $\mathrm{Hz}$, triple that of the large-scale prototype. When the wires are configured in the distributed Active Velcro surface, the effective bandwidth is increased to approximately $0.55 \mathrm{~Hz}$. Again, this gain is not due to the phased activation approach. Instead, an SMA wire is still energized on sequential steps, but is only partially cooled between steps. Using the phased activation approach, speeds as high as $5 \mathrm{~mm} / \mathrm{s}$ may be obtained.

\section{Acknowledgments}

This paper is based upon research supported the Air Force Laboratory under contract number F29601-98-D0210, and the Universities Space Research Association under subcontract number 9500-22.

\section{Conclusions}

This paper describes the proof-of-concept study of a new smart attachment mechanism called Active Velcro. Experimental testing has verified that the Active Velcro surface is capable of full planar translation and 360degree rotation with translation speeds of up to 3.7 $\mathrm{mm} / \mathrm{s}$ on the large-scale prototype and $2.8 \mathrm{~mm} / \mathrm{s}$ on the reduced-scale prototype.

The Active Velcro surface as a whole is capable of generating motion at a faster rate than the individual actuators that comprise the surface. Utilizing a redundant system of SMA actuators, an effective increase in the surface bandwidth of up to three-fold was observed when compared to the bandwidth of the individual SMA wires. This response is due to the fact that the wires are not required to fully cool before a guest surface post can be passed between individual prong groupings (translation increments).

While the current state-of-the-art active surfaces can generate planar motion, surface retention capabilities are unique to Active Velcro. This capability will expand the envelope of possible applications beyond those which operate in stable non-moving environments experiencing gravitational forces. For example, automated part sorting and materials handling systems may be designed to operate in the low gravity environment of space, the turbulent underwater environment, and aboard moving ground, sea or air vehicles.

\section{References}

${ }^{1}$ Suh, J.W., Darling, R.B., Bohringer, K.F., Donald, B.R., Baltes, H., Kovacs, G.T.A., 1999, "CMOS Integrated Ciliary Actuator Array as a General-Purpose Micromanipulation Tool for Small Objects," Journal of Microelectromechanical Systems, Dec, Vol. 8, No. 4, pp $483-496$.

${ }^{2}$ Ebefors, T., Mattsson, J.U., Kalvesten, E., Stemme, G., 1999, "A Robust Micro Conveyer Realized by Arrayed Polyimide Joint Actuators," in Proc. of the IEEE International Conference on Micro Electro Mechanical Systems, Jan. 17-21, pp 576 - 581 .

${ }^{3}$ Ataka, M., Omodaka, A., Takeshima, N., Fujita, H., 1993, "Fabrication and Operation of Polyimide Bimorph Actuators for a Ciliary Motion System," Journal of Microelectromechanical Systems, Dec, Vol. 2, No. 4, pp 146 - 150.

${ }^{4}$ Langlet, P., Collard, D., Akiyama, T., Fujita, H., 1997, "A Quantitative Analysis of Scratch Drive Actuation for Integrated X/Y Motion System," International Conference on Solid State Sensors and Actuators, June 16-19, Vol. 2, pp $773-776$.

${ }^{5}$ Iizuka, T., Fujita, H., 1997, "Precise Positioning of a Micro Conveyor Based on Superconducting Magnetic Levitation," in Proc. of the IEEE International Symposium on Micromechatronics and Human Science, Oct. 5-8, pp $131-135$.

${ }^{6}$ Nakazawa, H., Watanabe, Y., Morita, O., Edo, M., Yonezawa, E., 1997, "The Two-Dimensional Micro Conveyer: Principles and Fabrication Process of the 
Actuator," International Conference on Solid State Sensors and Actuators, June 16-19, Vol. 1, pp $33-36$.

${ }^{7}$ Konishi, S., Fujita, H., 1994, “A Conveyance System Using Air Flow Based on the Concept of Distributed Micro Motion Systems," Journal of Microelectromechanical Systems, June, Vol. 3, No. 2, pp $54-58$.

${ }^{8}$ Bohringer, K.F., Donald, B.R., Mihailovich, R., MacDonald, N.C., 1994, "A Theory of Manipulation and Control for Microfabricated Actuator Arrays," in Proc. of the IEEE Workshop on Micro Electro Mechanical Systems, Jan. 25-28, pp $102-107$.

${ }^{9}$ Bohringer, K.F., Donald, B.R., MacDonald, N.C., 1999, "Programmable Force Fields for Distributed Manipulation, with Applications to MEMS Actuator Arrays and Vibratory Parts Feeders," International Journal of Robotics Research, Feb., Vol. 18, No. 2, p $168-200$.

${ }^{10}$ Tadokoro, S., Fuji, S., Fushimi, M., Kanno, R., Kimura, T., Takamori, T., Oguro, K., 1998, "Development of a Distributed Actuation Device Consisting of Soft Gel Actuator Elements," in Proc. of the IEEE Conference on Robotics and Automation, Leuven, Belgium, May 16-20, Vol. 3, pp 2155 - 2160 .
${ }^{11}$ Yim, M, Duff, D.G., Roufas, K.D., 2000, "PolyBot: a Modular Reconfigurable Robot," in Proc. of the IEEE Conference on Robotics and Automation, San Francisco, CA, April 24-28, Vol. 1, pp 514- 520.

${ }^{12}$ Luntz, J.E., Messner, W., and Choset, H., 2000, "Closed-Loop Operation of Actuator Arrays," in Proc. of the IEEE Conference on Robotics and Automation, San Francisco, CA, April 24-28, Vol. 4, pp $3666-$ 3672 .

${ }^{13}$ Luntz, J.E., Messner, W., and Choset, H., 1999a, "Discrete Actuator Array Vectorfield Design for Distributed Manipulation," in Proc. of the IEEE Conference on Robotics and Automation, Detroit, MI, May 10-15, Vol. 3, pp 2235 - 2241.

${ }^{14}$ Luntz, J.E., Messner, W., and Choset, H., 1999b, "Open-Loop Orientability of Objects on Actuator Arrays," in Proc. of the IEEE Conference on Robotics and Automation, Detroit, MI, May 10-15, Vol. 3, pp 2242 - 2248.

${ }^{15}$ Bohringer, K.F., Suh, J.W., Donald, B.R., Darling, R.B., Kovacs, G.T.A., 1997, "Vector Fields for Tasklevel Distributed Manipulation: Experiments with Organic Micro Actuator Arrays," in Proc. of the IEEE Conference on Robotics and Automation, Albuquerque, NM, April 20-25, Vol. 2, pp 1779 - 1786. 